\title{
Adiabatic Low-Pass J Filters for Artifact Suppression in Heteronuclear NMR
}

Meier, Sebastian; Benie, Andrew J; Duus, Jens Øllgaard; Sørensen, Ole W.

Published in:

ChemPhysChem

Link to article, DOI:

$10.1002 /$ cphc. 200900072

Publication date:

2009

Link back to DTU Orbit

Citation (APA):

Meier, S., Benie, A. J., Duus, J. Ø., \& Sørensen, O. W. (2009). Adiabatic Low-Pass J Filters for Artifact

Suppression in Heteronuclear NMR. ChemPhysChem, 10(6), 893-895. https://doi.org/10.1002/cphc.200900072

\section{General rights}

Copyright and moral rights for the publications made accessible in the public portal are retained by the authors and/or other copyright owners and it is a condition of accessing publications that users recognise and abide by the legal requirements associated with these rights.

- Users may download and print one copy of any publication from the public portal for the purpose of private study or research.

- You may not further distribute the material or use it for any profit-making activity or commercial gain

- You may freely distribute the URL identifying the publication in the public portal

If you believe that this document breaches copyright please contact us providing details, and we will remove access to the work immediately and investigate your claim. 


\title{
Adiabatic Low-Pass J Filters for Artifact Suppression in Heteronuclear NMR
}

\author{
Sebastian Meier, ${ }^{[b]}$ Andrew J. Benie, ${ }^{[c]}$ Jens $\varnothing$. Duus, ${ }^{[b]}$ and Ole W. Sørensen ${ }^{*[a]}$
}

Adiabatic pulses that consist of frequency-swept radio-frequency ( $r f$ irradiation in the presence of a static magnetic field gradient have many applications in NMR spectroscopy. The prime feature of such pulses is the very effective and uniform inversion of magnetization over large bandwidths at low radio-frequency power-something that is unmatched by other types of conventional or composite pulses. ${ }^{[1,2]}$

Applications of adiabatic pulses include multipulse decoupling sequences and several multidimensional experiments where broadband inversion is critical in order to refocus evolution under chemical shifts. Another application is for suppression of homonuclear zero-quantum coherence (ZQC) in nuclear Overhauser effect spectroscopy (NOESY) and total correlation spectroscopy (TOCSY) experiments, which is quite successful except for the smallest ZQC frequencies..$^{[3]}$ The ZQC suppression scheme applies a frequency-swept pulse in the presence of a pulsed field gradient. As a result, individual elements of the sample are subject to different effective chemical shift evolution times so that subsequent averaging over the sample results in cancellation of ZQC artifacts. ${ }^{[3]}$

Herein, we extend the use of adiabatic inversion pulses to the suppression of unwanted correlations or artifacts which obscure heteronuclear multidimensional NMR spectra of medium-size organic molecules or natural products. Such spectra may suffer from complexity and overlap at increasing molecular weight, low purity and low chemical shift dispersion. Indeed, overlap and ambiguities can be particularly severe where chemical-shift dispersion is small, such as in carbohydrates. Low-pass J filters (LPJFs) are crucial for suppression of one-bond correlations in all types of long-range correlation spectra, but unfortunately, they often fail in tightly coupled spin systems. $^{[4,5]}$

One application of our novel adiabatic filter is as a remedy for the shortcomings of conventional LPJFs in, for example, heteronuclear ${ }^{1} \mathrm{H}-{ }^{13} \mathrm{C}$ multiple-bond correlation $(\mathrm{HMBC}) .{ }^{[5,6]}$ It uses an adiabatic pulse to introduce heterogeneity for evolution under ${ }^{13} \mathrm{C}-{ }^{1} \mathrm{H}$ scalar or residual dipolar couplings by means of a frequency-swept $180^{\circ}$ pulse on ${ }^{13} \mathrm{C}$ that inverts ${ }^{13} \mathrm{C}$ nuclei at different positions in the NMR sample at different

[a] Prof. O. W. Sørensen

DTU Chemistry, Technical University of Denmark Kemitorvet 207, DK-2800 Kgs. Lyngby (Denmark)

Fax: (+45) 4588-3136

E-mail:ows@kemi.dtu.dk

[b] Dr. S. Meier, Prof. J. Ø. Duus

Carlsberg Laboratory

Gamle Carlsberg Vej 10, DK-2500 Valby (Denmark)

[c] Dr. A. J. Benie

Novo Nordisk A/S

Diabetes Protein Engineering, DK-2760 Måløv (Denmark) times (Figure 1). If the $180^{\circ}$ pulse occurs at time zero at the top and at the end of the adiabatic pulse of length $2 \tau_{2}=1 \mathrm{~J}$ at the bottom, then the evolution under the coupling varies from

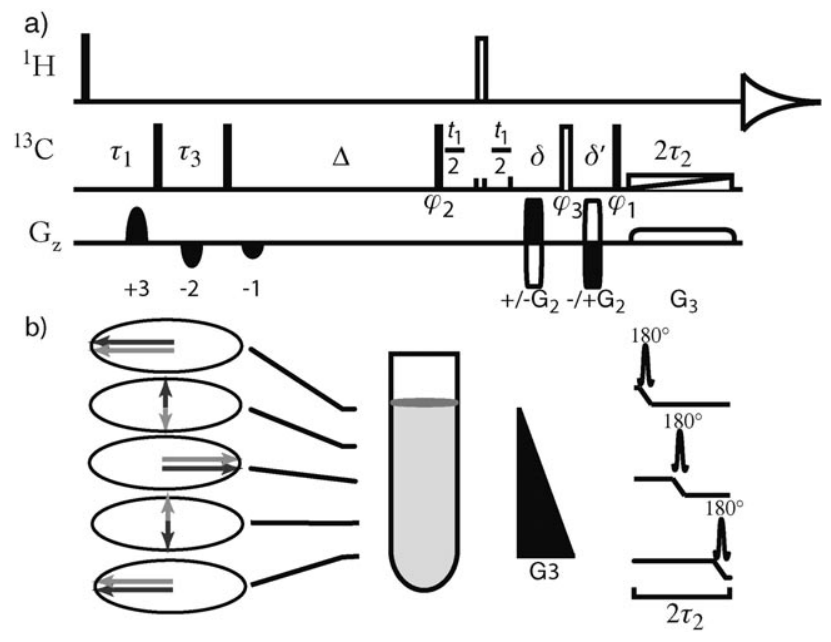

Figure 1. Pulse sequence for the clean $\mathrm{HMBC}$ experiment with an initial second-order and terminal adiabatic low-pass $J$ filter. Recommended delays for the filters are $\tau_{1}=1 / 2\left[{ }^{1} \mathrm{~J}^{\mathrm{min}}+0.07\left({ }^{1} \mathrm{~J}^{\mathrm{max}}-{ }^{1} \mathrm{~J}^{\mathrm{min}}\right)\right]^{-1}, \tau_{2}=\left({ }^{1} \mathrm{~J}^{\mathrm{max}}+{ }^{1} \mathrm{~J}^{\mathrm{min}}\right)^{-1}$ and $\tau_{3}=1 / 2\left[\left[^{1} J^{\mathrm{min}}-0.07\left({ }^{1} J^{\mathrm{max}}-{ }^{1} J^{\mathrm{min}}\right)\right]^{-1} \cdot{ }^{[7]} \delta\right.$ and $\delta^{\prime}$ are gradient delays, where $\delta^{\prime}=\delta+6 \mu$ s accounts for the delay of the first point in the ${ }^{13} \mathrm{C}$ dimension. The integrals over each gradient pulse $G_{2}$ is $\gamma_{H} / 2 \gamma_{C}$ times the integral over gradient $G_{3}$ in order to achieve coherence selection. The recommended phase cycle is $\phi_{1}=\{\mathrm{x},-\mathrm{x},-\mathrm{x}, \mathrm{x}\}, \phi_{2}=\{\mathrm{x}, \mathrm{x}, 4(-\mathrm{x}), \mathrm{x}, \mathrm{x}\}$ and

$\phi_{3}=\{4(x), 4(y), 4(-x), 4(-y)\}$ with the receiver phase $\{x,-x\}$. The dephasing under one-bond couplings during the adiabatic filter by spatially dependent application of the $180^{\circ}{ }^{13} \mathrm{C}$ pulse is shown in (b).

0 to $2 \pi$ across the sample volume at the end of $2 \tau_{2}$ and hence cancellation will occur at the end of the filter (Figure $1 \mathrm{~b}$ ). Explicitly, the attenuation factor for a heteronuclear doublet amounts to $\sin \left(2 \pi J \tau_{2}\right) / 2 \pi J \tau_{2}$. Thus, suppression of ${ }^{1} \mathrm{H}$ magnetization one-bond coupled to a ${ }^{13} \mathrm{C}$ nucleus occurs for $2 \tau_{2}=$ $\left\langle{ }^{1} \mathrm{~J}_{\mathrm{CH}}\right\rangle^{-1}$ whilst long-range coupled ${ }^{1} \mathrm{H}$ magnetization is virtually unaffected by the filter. This filter is robust with respect to pulse miscalibration and operates efficiently without the need to cycle the phases of pulses that otherwise is a common feature of non-destructive LPJFs. ${ }^{[5,6]}$

We illustrate the novel adiabatic low-pass J filter by an application to $\mathrm{HMBC}$ in its recently introduced clean version. ${ }^{[5]}$ Clean $\mathrm{HMBC}$ is designed for suppression of strong-coupling-induced artifacts that can obscure genuine long-range correlations and possibly lead to incorrect spectral assignments. These artifacts arise from incomplete suppression of one-bond ${ }^{1} \mathrm{H}$ magnetization by conventional initial LPJFs and additional coherence transfers by pulses in the course of the pulse sequence in strongly coupled spin systems. The adiabatic LPJF at the end 
of the pulse sequence suppresses these strong-coupling-induced artifacts.

If the adiabatic LPJF were the only means of suppressing one-bond coupled ${ }^{1} \mathrm{H}$ magnetization, the suppression would be inadequate, as this filter is effectively of first order. ${ }^{[4]}$ Therefore, the pulse sequence in Figure 1 is designed with two conventional LPJF steps at the beginning of the sequence and one terminal adiabatic filter in such a way that the three filtering steps together amount to an effective overall third order LPJF.

Figure 2 shows third-order filtered $\mathrm{HMBC}$ spectra of raffinose [ $\alpha$-D-galactopyranosyl- $(1 \rightarrow 6)-\alpha$-D-glucopyranosyl-( $1 \rightarrow 2)-\beta$-Dfructofuranose] recorded without and with the adiabatic LPJF element. 1D sections from the 2D spectra are shown in Figure $2 \mathrm{~b}$. Clearly, artifacts are present in the standard HMBC spectrum for all three moieties, fructose, glucose and galactose, whilst they are strongly attenuated $(>90 \%)$ by the adiabatic LPJF. Due to the low chemical shift dispersion of carbohydrates, resonances for non-anomeric protons and carbons in oligosaccharides are in a highly crowded spectral region for complex oligosaccharides and similar problems are common for a variety of medium-size organic compounds with molecular masses of few $\mathrm{kDa}$. The presence of one-bond artifacts split along the proton dimension by ${ }^{1} \mathrm{~J}_{\mathrm{CH}}$ exacerbates spectral crowding for these compounds. This is where the adiabatic LPJF comes in handy. Figure 3 shows the application to a

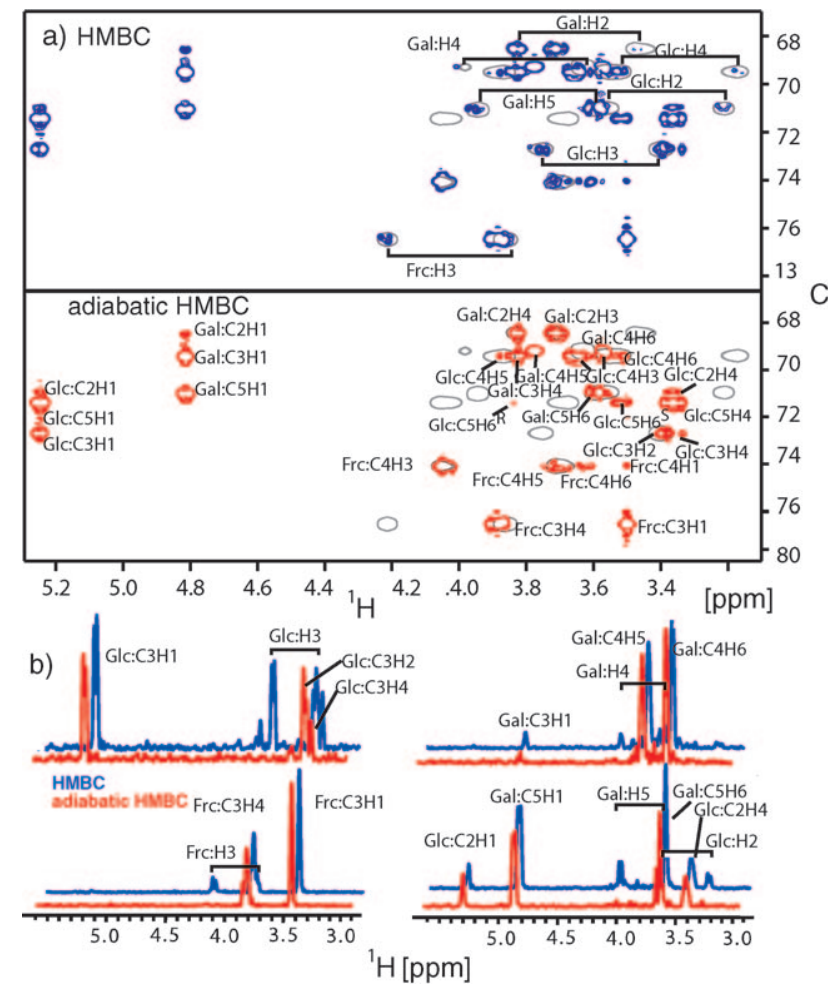

Figure 2. a) Third-order LPJF HMBC spectra recorded without (blue) and with (red) the novel adiabatic filter of $50 \mathrm{~mm}$ raffinose in $\mathrm{D}_{2} \mathrm{O}$ plus an overlaid coupled HSQC spectrum (grey) indicating the positions of strong-coupling-induced artifacts. b) $1 \mathrm{D}$ HMBC sections from the ${ }^{13} \mathrm{C}$ frequencies of $C_{4}$ (galactose), $C_{3}$ (glucose), $C_{3}$ (fructose) and $C_{5}$ (galactose). Use of the adiabatic filter allows the spectra to be recorded with only two scans per increment.

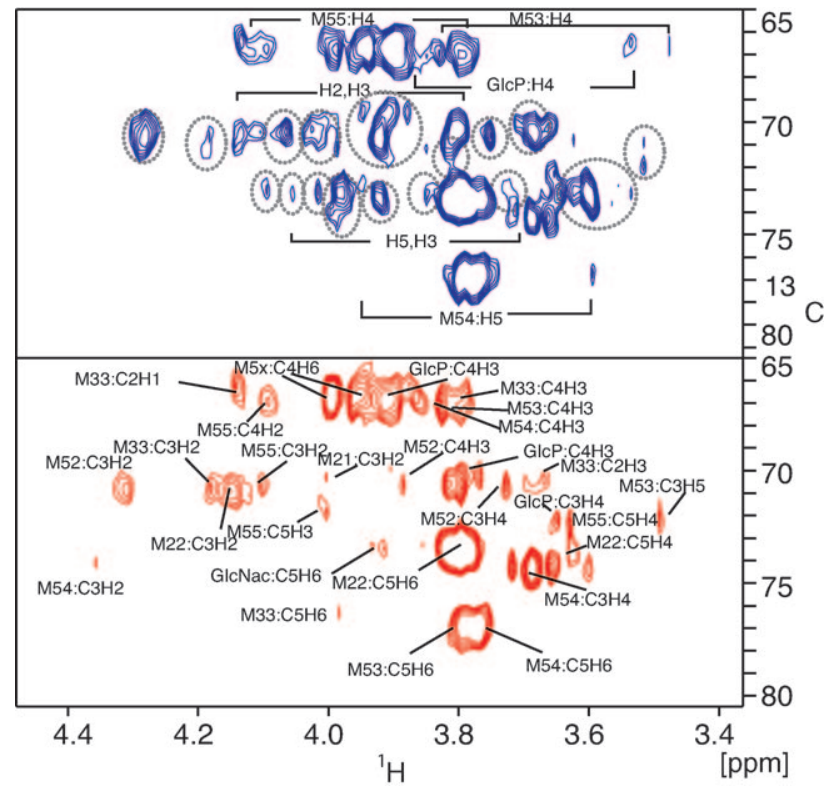

Figure 3. HMBC spectra without (blue) and with (red) the adiabatic LPJF of $3 \mathrm{mg}$ Pichia pastoris mannan dissolved in $600 \mathrm{~mL}^{2} \mathrm{H}_{2} \mathrm{O}$. Artifacts are encircled with grey dotted lines.

mannan preparation from the yeast Pichia pastoris (Figure 3). ${ }^{[10]}$ While artifacts clearly render the resonance assignment in the standard HMBC spectrum extremely challenging, the adiabatic filter effectively purges artifacts and thus reduces ambiguity.

In summary, we have introduced a novel adiabatic low-pass $J$ filter and shown an application for artifact suppression in heteronuclear multiple-bond correlation NMR. This purging of artifacts reduces ambiguity and is crucial in crowded spectra. The minimum instrument time compared to the earlier clean HMBC scheme is reduced by a factor of four by the adiabatic LPJF. ${ }^{[5]}$

Earlier works have made use of schemes to generate spatially dependent shift evolution for fairly different applications. ${ }^{[3,8,9]}$ Our novel adiabatic element might also find application in this realm of ultrafast multidimensional NMR spectra ${ }^{[8]}$ as a means of generating a coupling dimension in a time-effective manner.

\section{Experimental Section}

All NMR experiments were performed on a Bruker DRX 400 spectrometer. Raffinose was dissolved in $\mathrm{D}_{2} \mathrm{O}(600 \mu \mathrm{L})$ to a final concentration of $50 \mathrm{~mm}$ without further adjustment of $\mathrm{pH}$ or ionic strength. Mannan from the yeast Pichia pastoris was purified in ${ }^{13} \mathrm{C}$ isotope enriched form as decribed previously. ${ }^{[10]}$ The mannan ( $3 \mathrm{mg}$ ) was dissolved in $\mathrm{D}_{2} \mathrm{O}(600 \mu \mathrm{L})$ without further adjustments. NMR spectra of raffinose and Pichia pastoris mannan were recorded at $30^{\circ} \mathrm{C}$ and $50^{\circ} \mathrm{C}$, respectively. Both chirp ${ }^{[1]}$ and WURST (wideband, uniform rate, and smooth truncation ${ }^{[2]}$ pulses were tested for use in the swept $180^{\circ}$ pulse/gradient pair. Results were comparable and spectra shown in Figures 2 and 3 were recorded with $180^{\circ}$ chirp pulses. Adiabatic pulses were swept through $25 \mathrm{kHz}$ in $6.78 \mathrm{~ms}\left[2\left(J_{\min }{ }^{\mathrm{CH}}+J_{\max }{ }^{\mathrm{CH}}\right)^{-1}\right.$, with $\left.J_{\min }{ }^{\mathrm{CH}}=125 \mathrm{~Hz}, J_{\max }{ }^{\mathrm{CH}}=170 \mathrm{~Hz}\right]$, with a maximum field strength $\gamma \mathrm{B}_{1}$ of $1.7 \mathrm{kHz}$. The gradient pulse that is applied simultaneously with the adiabatic pulse was applied 
at $20 \%$ of the maximum gradient strength $\left(60 \mathrm{G} \mathrm{cm}^{-1}\right)$ as a rectangular shape. $G_{2}$ was applied as a rectangular pulse of $3 \mathrm{~ms}$ duration with $90.25 \%$ of the maximum gradient strength. The strong gradients for coherence selection and adiabatic low-pass J filter were followed by recovery delays of $200 \mu$ s to minimize eddy-current-induced artifacts. The heteronuclear multiple-bond correlation spectra of raffinose shown in Figure 2 were recorded as data matrices of $1024\left({ }^{1} \mathrm{H}\right) \times 128\left({ }^{13} \mathrm{C}\right)$ complex points with total acquisition times of $256\left({ }^{1} \mathrm{H}\right)$ and $25\left({ }^{13} \mathrm{C}\right) \mathrm{ms}$, while HMBC spectra of the mannan as shown in Figure 3 were recorded as data matrices of $2048\left({ }^{1} \mathrm{H}\right) \times 32$ $\left({ }^{13} \mathrm{C}\right)$ complex points with total acquisition times of $512\left({ }^{1} \mathrm{H}\right)$ and 6 $\left({ }^{13} \mathrm{C}\right) \mathrm{ms}$. The spectra without the adiabatic LPJF employed an initial 3rd order LPJF using the same $\tau_{1}, \tau_{2}, \tau_{3}$ delays.

Keywords: analytical methods - artifact suppression heteronuclear multiple-bond correlation - low-pass J filter • NMR spectroscopy
[1] J. M. Bohlen, G. Bodenhausen, J. Magn. Reson. Ser. A 1993, 102, 293301.

[2] E. Kupce, R. Freeman, J. Magn. Reson. Ser. A 1995, 115, 273-276.

[3] M. J. Thrippleton, J. Keeler, Angew. Chem. 2003, 115, 4068-4071; Angew. Chem. Int. Ed. 2003, 42, 3938-3941.

[4] H. Kogler, O. W. Sørensen, G. Bodenhausen, R. R. Ernst, J. Magn. Reson. 1983, 55, 157-163.

[5] P. Wurtz, P. Permi, N. C. Nielsen, O. W. Sørensen, J. Magn. Reson. 2008, 194, 89-98.

[6] A. Meissner, O. W. Sørensen, Magn. Reson. Chem. 2000, 38, 981-984.

[7] O. W. Sørensen, S. Dønstrup, H. Bildsøe, H. J. Jakobsen, J. Magn. Reson. 1983, 55, 347-354.

[8] L. Frydman, T. Scherf, A. Lupulescu, Proc. Natl. Acad. Sci. USA 2002, 99, $15858-15862$

[9] T. Schulte-Herbrüggen, Angew. Chem. 2003, 115, 5427-5429; Angew. Chem. Int. Ed. 2003, 42, 5270-5272.

[10] E. Vinogradov, B. O. Petersen, J. O. Duus, Carbohydr. Res. 2000, 325, 216-221.

Received: February 2, 2009

Published online on March 13, 2009 